\title{
Position And Vision Information of Relative Vehicle's Using Procrustes Analysis Method for Position Correction
}

\author{
I.S Weon ${ }^{1}$, S.G Lee ${ }^{2 *}$, S.C Moon ${ }^{3}$ \\ ${ }^{1}$ Department of Mechanical Engneering Graduate School, KyungHee University, \\ YongIn, 17104, Korea \\ ${ }^{2}$ Departmentt of Mechanical Engneering School, KyungHee University, YongIn, \\ 17104, Korea \\ sglee@khu.ac.kr \\ ${ }^{3}$ Core Tech R\&D Lab., LIGNexl, SeongNam 13488, Korea
}

\begin{abstract}
This paper proposes a vehicle-positioning improvement algorithm that utilizes positioning data collected from budget GPS devices and vehicle data obtained from ODB devices and relays the information via WAVE communications between cars. Data from both sender and recipient vehicles are matched with Procrustes analysis based on the location in-formation of the other car. The corrected location data is used in order to calculate the distance be-tween the two cars. By using commercial lane-detection technology, the distance between the cars and each lane can also be calculated with the calibrated location data. The difference between the cars' video-estimated distance and GPS data can be used to improve vehicle-location correction algorithms.
\end{abstract}

Keywords: Wireless Access in Vehicle Environment, Vehicle to Vehicle Communication, GPS Precision Positioning, ITS, Procrustes Analysis

\section{Introduction}

Accurately estimating a car's position with GPS technology is a necessity in vehicle technology such as self-driving cars and Advanced Driver Assistance Systems (ADASs).[1] When a car is able to pinpoint its exact position, it can improve the car's perception of its environment, which leads to better controls based thereon. Better controls also mean safety and fuel economy will be improved. [13]

The Global Navigation Satellite System (GNSS), the most used method in positioning technology, is known to provide real-time positioning data with an accuracy of tens of meters using C/A (Coarse/Acquisition) code data from a single receiver. [4] However, the need for a more accurate positioning technology is rising; accordingly many research projects are attempting to combine various sensors to improve positioning accuracy. In particular, MEMS technology has seen much improvement, and various studies conducted on the combination of budget MEMS IMUs and GPS systems have enabled stable vehicle positioning data in regions with poor GPS signals. [5] However, due to the low quality of MEMS IMUs, the positioning error starts to grow exponentially when GPS signals are inaccessible, resulting in issues in reliability.

Many studies implementing video and image sensors have been conducted in order to solve the positioning error in GPS/IMU systems. The combination of such sensors inevitably increased the size of the sensor device. This resulted in the sensors being difficult to install, which narrowed the target vehicles down to newly developed smart cars. In order to alleviate this issue, cooperative positioning correction methods through car-to-car and car-to-infrastructure communication have been developed. 
With the development of vehicular communication technologies, there has been many research and technologies on vehicular communications between multiple cars (V2V) and infrastructures (V2I) by sharing positioning information and the relative distance measured by wireless signals to improve cooperative navigation systems.[9] Studies have attempted to improve positioning accuracy of a car with data collected from itself and another car. A Kalman filter is used in order to estimate the location of another car. By using that estimated positioning data as a baseline, the driver's car uses a baseline tracking filter in order to improve its positioning accuracy. However, this technology relies heavily on another car providing its positioning information, which means that the performance of the positioning accuracy is directly correlated with the reliability of the data received. In addition, Hybrid-Sum Product Algorithm over Wireless Network (HSPAWN) technology, which implements a simple calculation process that provides an optimal solution based on GNSS positioning technology, improves positioning accuracy in low-coverage areas.[9] However, limitations exist, as the wireless signal measurement sensor (UWB module) is not attached to current-market vehicles. In order to provide a more widespread solution, there has been an increase in using WAVE communication methods that can allow communications with other cars and infrastructures, even during high-speed travel.[11] The recent Vehicle Convergence Technology Development Project between BMW and KETI shows how WAVE communication is utilized in C-ITS technologies. WAVE communication technology linked with UTIS is also showing how inter-car and inter-infrastructure communication is moving towards a standard. [10]

This paper proposes a vehicle positioning improvement algorithm that utilizes positioning data collected from budget GPS devices and vehicle information obtained via OBD devices through WAVE communication between cars. Cars were lined up in single file and used video information along with lane spacing distances to experiment with the positioning correction algorithm. [1]

\section{Obtaining Information from a Nearby Car}

\subsection{Predicting a Distance between Two Cars by Vision Information}

Video data on the relative distance of cars and lanes were collected using PLK's LDWS module in 100-ms intervals. [1]

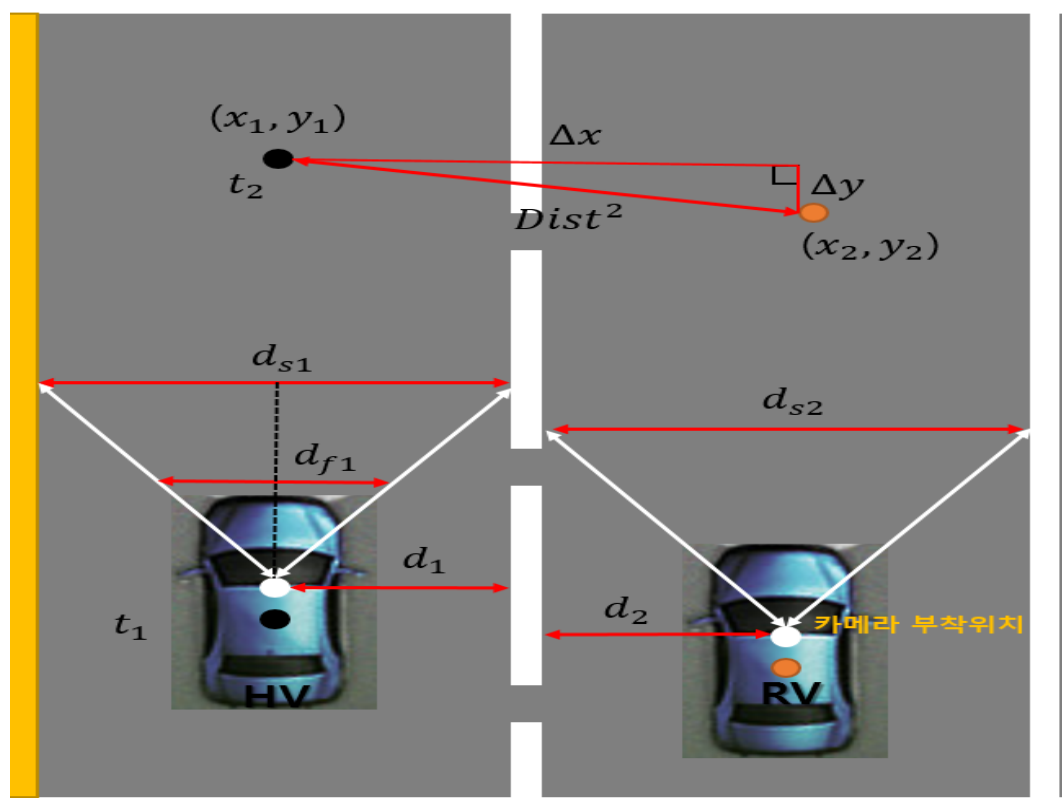

Figure 1. Calculation of a Relative Distance by Roadscope LX 
At the initial state, the cars on two lanes are positioned as shown Figure. 1, and the relative distance between cars and lanes are calculated by comparing the initial distance to the measured space on the left and right of each car. As described in Figure 1, the car's lane distance, $d_{l}$, is calculated with the video data from the front of the car, $d_{s l}$, the lane widths ratios detected from videos on the grill of the car, $d_{f 1}$, and the distance ratio to the module's location. Thus, cars at location $t_{l}$ utilize the collected data from the video module to calculate the distance between the lanes $d_{1}$ and $d_{2}$. This information is then used in $d_{1}+d_{2}=d_{v}$ to calculate the distance between two cars

\subsection{Estimating Vehicle Distances and Angles using WAVE Information}

2.2.1. Obtaining Vehicle Information via WAVE Communication: V2V communication utilizes the WAVE protocol to send and receive vehicle information. The WAVE protocol structure consists of multiple IEEEs standards, IEEE 1609.3, 1609.4, and 802.11p, for PHY and MAC protocols.[11]

This research utilizes a periodic BSM (Basic Safety Message), defined in SAE (Society of Automotive Engineers) J2735 as WSM (WAVE Short Message), to communicate positioning and vehicle information from other cars via WAVE communications. The information is used in the proposed positioning correction algorithm, which includes speed data, positioning data, and directional angles obtained from OBUs

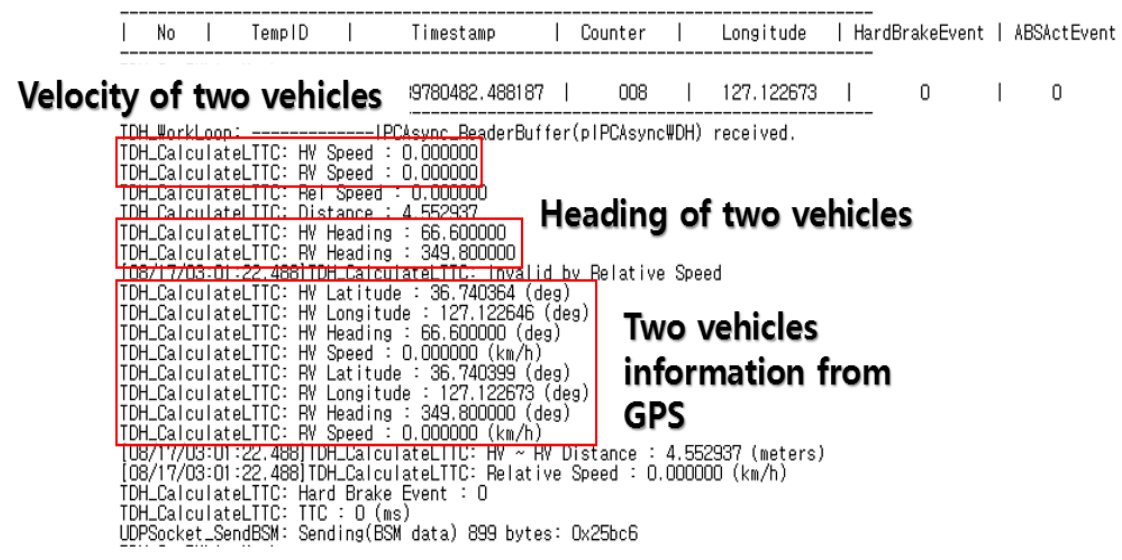

Figure 2. Position and Vehicle Information received by WAVE

2.2.2. Converting GPS Information to $X, Y$ Coordinates: In order to implement GIS lane latitude and longitude data obtained from GPS to the system, a conversion to a two-dimensional coordinate system is required. This has been completed using TM projection utilizing the Gauss-Kruger projection technique.[3]

$$
\begin{gathered}
A=\left(\lambda-\lambda_{0}\right) \cos \Phi \\
Y(E)=\Delta Y+k_{0} N\left\{A+\frac{A^{3}}{6}(1-T+C)+\frac{A^{5}}{120}\left(5-18 T+T^{2}+72 C-58 e^{2}\right)\right\} \\
X(N)=\Delta Y+k_{0} N\left\{A+\frac{A^{3}}{6}(1-T+C)+\frac{A^{5}}{120}\left(5-18 T+T^{2}+72 C-58 e^{2}\right)\right\}
\end{gathered}
$$


In $T-\tan ^{2} \Phi, C=\frac{e^{2}}{1-e^{2}} \cos ^{2} \Phi, e^{2}$ is the first eccentricity, $e^{\prime 2}$ is the second eccentricity, $a$ is the longer radius of the ellipsoid, $b=a(1-f)$ is the shorter radius of the ellipsoid, $f$ is the ellipsoid's flatness applied by the WGS 84 Ellipsoid. The curvature radius based on latitude is obtained by Equation (4).

$$
N=\frac{a}{\sqrt{a-e^{2} \sin ^{2} \Phi}}
$$

The following TM projection method can be applied to change the latitude and longitude information to an X, Y coordinate system, and then can be used in the positioning correction algorithm.

2.2.3. Estimated Relative Distance and Heading by GPS Coordinate: When $x, y$ coordinates of vehicles are available, the relative distance and direction angle can be calculated. The process for calculating the location on the two-dimensional plane utilizing TM projection is used for obtaining the positioning information of the driver's car. Here, the absolute position difference with the universal plane is represented by the direction angle, which should be established by the driver's car.

$$
\begin{array}{r}
\Delta x=x_{2}-x_{1} \\
\Delta y=y_{2}-y_{1} \\
\theta=\tan ^{-1}\left(\frac{\Delta y}{\Delta x}\right) \\
\beta=\alpha-\theta \\
\Delta x^{\prime}=d \cdot \sin \beta \\
\Delta y^{\prime}=d \cdot \cos \beta
\end{array}
$$

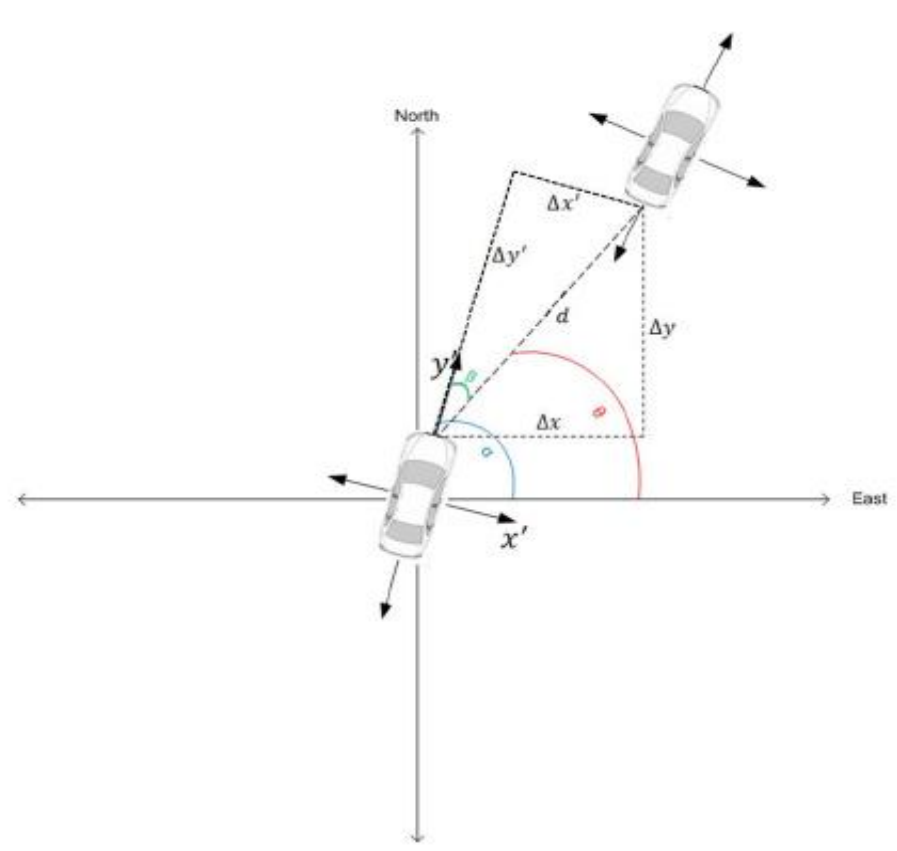

Figure 3. Relative Distance Measurement 
Accordingly, as mentioned in Equation (7), each car has a horizontal axis indicated by $\Delta \chi$. In addition, each car can change its direction angle continuously, as seen in Equation (6), which must be updated continuously on the coordinate system. As shown in Figure. 3, the relative distance and direction angle is determined by where the other car is positioned.

\section{Improving Positional Data based On the Other Car's GPS Information}

\subsection{Procrustes Analysis}

Procrustes analysis was used in order to improve the driver's relative positional data based on another vehicle's driving path. Procrustes analysis is used to compare and match respective sections from two data sets in order to find matching corresponding points. This analysis method is mainly used in video and shape analysis, and its purpose is to find and match the most similar size, displacement, and rotation parameters from shapes with two or more boundary points, as shown in Figure. 4.

Procrustes analysis is applied using matrix A containing the driver vehicle's position data, and ( $\mathrm{p} \times \mathrm{k}$ ) matrix B containing the other vehicle's position data. Equations (8), (9), and (10) are used in order to obtain $j^{T}=[11 \ldots 1],(1 \times p)$ unit vector and scaling factor $\mathrm{c}$, rotation matrix $\mathrm{T}(\mathrm{k} \times \mathrm{k})$, and translation $\mathrm{t}(\mathrm{k} \times 1)$.

$$
\begin{gathered}
\frac{\partial F}{\partial T}=2 c^{2} A^{T} A T-2 c A^{T} B+2 c A^{T} j t^{T}+T\left(L+L^{T}\right)=0 \\
\frac{\partial F}{\partial T}=2 p t-2 B^{T} j+2 c T^{T} A^{T} j=0 \\
\frac{\partial F}{\partial T}=2 c t r T^{T} A^{T} A T-2 t r B^{T} A T+2 t r T^{T} A^{T} j t^{T}=0 \\
c=\frac{t r T^{T} A^{T}\left(I-\frac{j j^{T}}{p}\right) B}{t r A^{T}\left(I-\frac{j j^{T}}{p}\right) A} \\
t(B-c A T)^{T} j / p \\
T=A^{T}\left(I-\frac{j j^{T}}{p}\right) B
\end{gathered}
$$

The calculated scaling factor $c$, translation $t$, and $T$ are then used in order to obtain the configuration, $P_{V^{\prime} k}=c A T+j t^{T}-B$ this is then used in generating a matching matrix by calculating EOP (Extended Orthogonal Procrustes) on matrices $A$ and $B$. The obtained configuration, $P_{V}$, is used to obtain the current configuration, $P_{V^{\prime}}$. This research uses the above analysis by handling each $P_{V^{\prime}}$ element as positioning data for the constant matching and updating of $P_{V^{\prime}}$. 


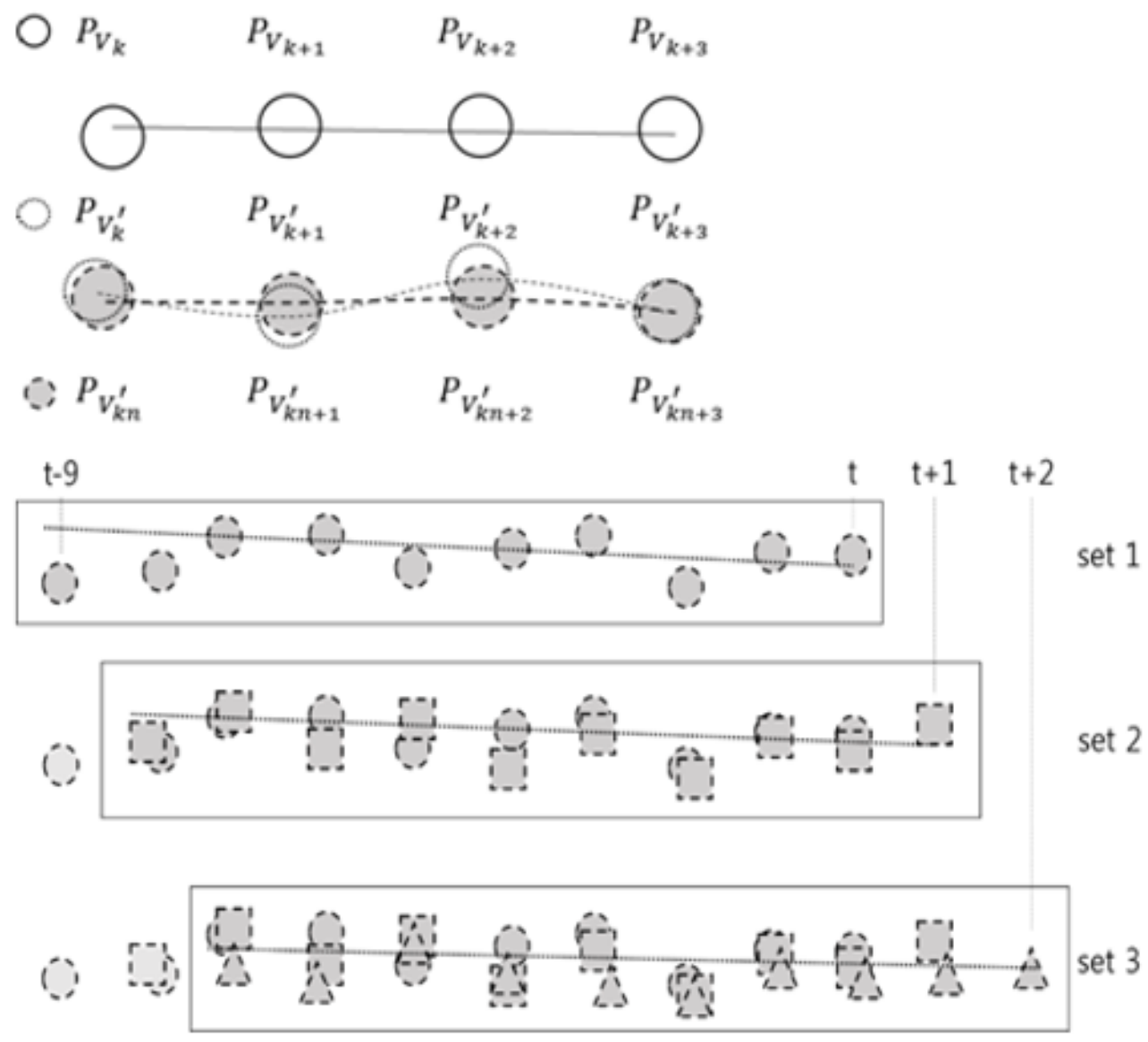

Figure 4. Application of Procrustes Analysis

$P_{V i}$ is the other car's position, $P_{V^{\prime}}$ is the driver's car's position, is the matching location obtained from the other vehicle's position. This research uses Procrustes analysis on the vehicles' route information to improve the relative positional accuracy. It was possible to improve the driver vehicle's positioning information by matching the less-reliable positional data of the driver with the more-reliable positional data of the nearby vehicle. The sampling interval for the route correction was the same as the collection interval of $100 \mathrm{~ms}$, and a total of 10 locations were matched. In order to provide continuity, each information dataset was overlapped on the previous one, as shown in Figure. 4.

\subsection{Minimizing Error Using Least-Squares Method}

When a matching procedure via Procrustes analysis is completed, a set of new coordinates are generated at each location instance. In order to obtain the coordinates with the least amount of errors, a recursive least-squares method was applied. The optimal approximation equations of each $n$ location value were obtained by using this method.

As shown in Figure. 5, in order to obtain the optimal approximation equation, the next $\mathrm{n}$ locational information sets were overlapped to the previous sets.

Based on the driver's car, the procedure of the least-squares method on each Procrustes analysis set is outlined in Figure. 5. In step 1, the least-squares method is applied on the region from $\mathrm{t}-9$ to $\mathrm{t}$; step 2 applies it on the combined regions of $\mathrm{t}-8$ to $\mathrm{t}+1$ and $\mathrm{t}-8$ to t. The same method is applied to all regions to obtain the approximation equation. After 
obtaining the closest location information to the approximation for each step, the set with the most reliable positioning information is generated

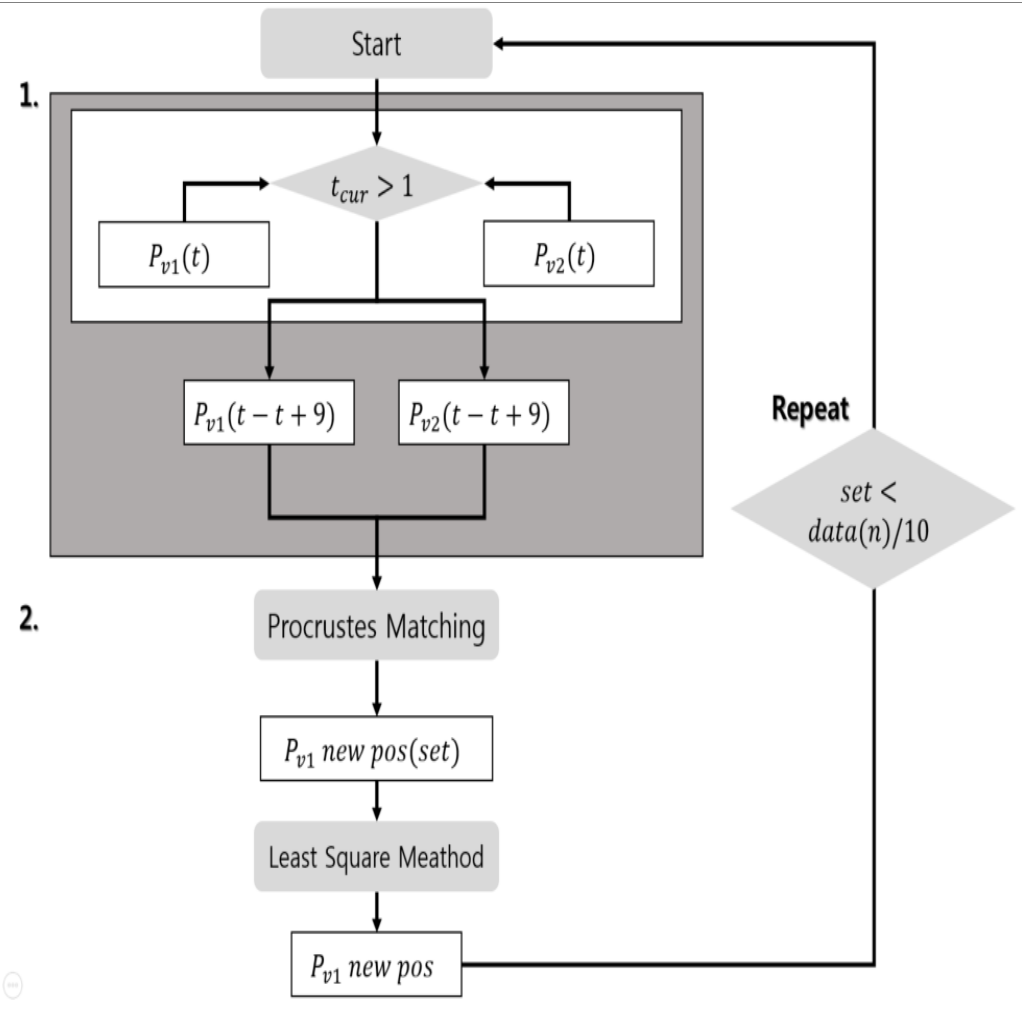

Figure 5. Extraction Step of Reliability Positioning Data Set

\subsection{Using Nearby-Vehicle Video Information to Improve Position Accuracy}

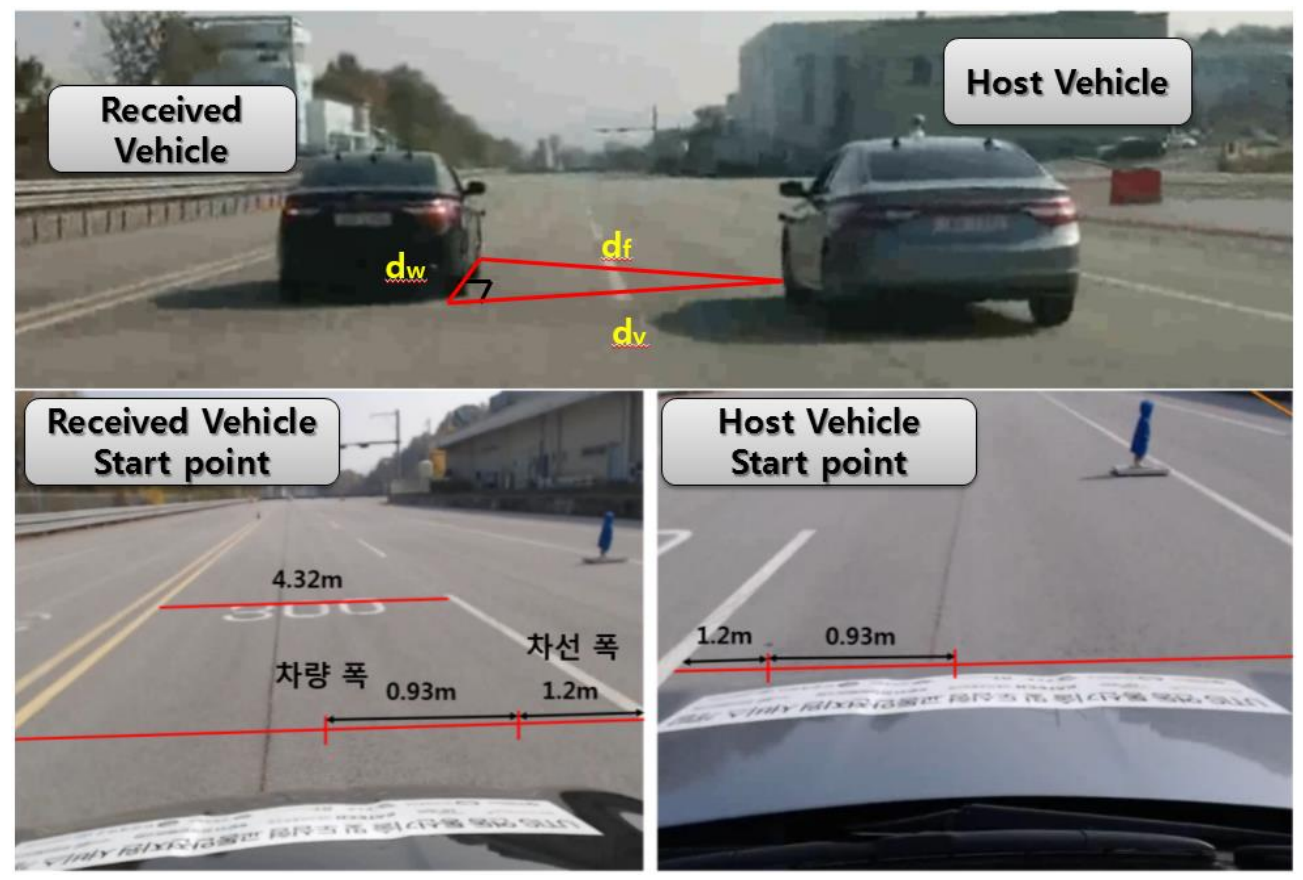

Figure 6. Two Vehicles Positioning on Field Lane 
The Procrustes-corrected positional data coordinates can be compared with the inter-car and inter-lane distance data obtained by video data.

Figure 6 shows the actual location and distance in relation to a nearby car. The full length of the nearby car can be set as $d_{w}$, and the distance between the two cars obtained by video can be represented as $d_{v}$. These two values allow for the calculation of the basis distance $d_{f}$. This distance obtained from video $d_{f}$ can then be compared with coordinate calculations Dist from before to further reduce the error $d_{f}-$ Dist $=\Delta e$ based on $t$

\section{Road Test Organization and Results}

\subsection{System Composition}

Experiments were carried out in a platform with actual cars. The WAVE communication antenna using IEEE 802.11p and 1690.4 communication protocols were attached to the back of the vehicles. As shown in Figure. 7, Novatel's low-cost 2.5maccuracy GPS modules were attached to the midsections of cars. The WAVE antennas were attached at the same height and $1,135 \mathrm{~mm}$ behind the GPS modules. In order to verify the positioning correction algorithm using nearby cars, experiments were conducted at a Car-Parts Research Center located in Cheonan-si, Poongse-myun, with a 900m-long and 50m-wide 2-lane test track

In our experiments, we set up a device which is high accuracy performance with Novatel GPS. And this device using for reference position data in this research. Received vehicle is attached low-cost GPS made by Trimble. So we use 3 GPS devices and compared with each test.

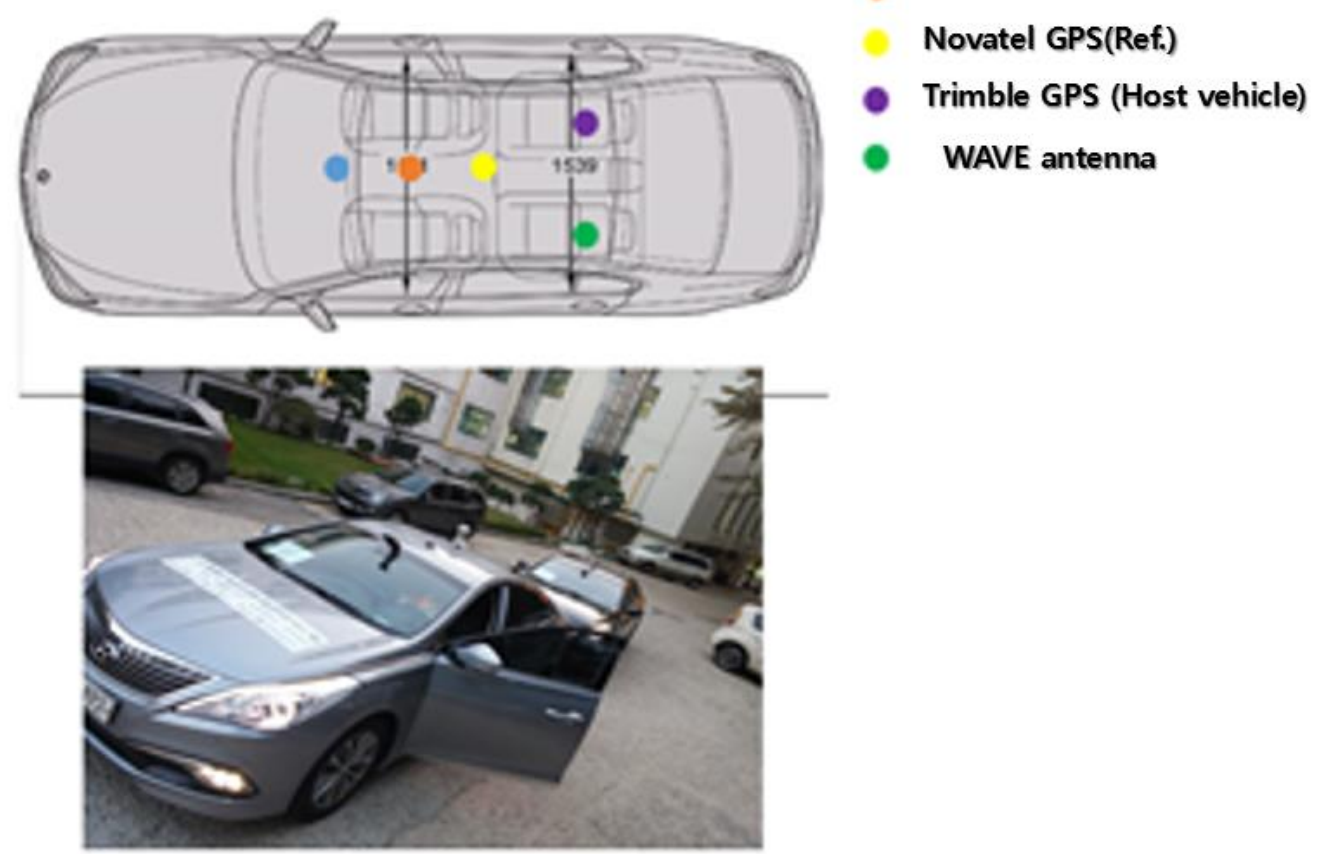

Figure 7. Sensor Position and Other Configuration of Test Vehicle 


\subsection{Experiment reSults}

4.2.1. Experimental Results of the Procrustes Analysis: In a straight road of $552 \mathrm{~m}$, two cars were positioned with 2-3 meters of space and were driven single file in speeds between $30 \mathrm{~km} / \mathrm{h}$ and $40 \mathrm{~km} / \mathrm{h}$. The GIS lane data were provided by the Research Center and were compared with the obtained data. A single Procrustes Analysis was used to match and improve the positional data between the two cars. As shown in Figure. 8, the correction data is expressed when the basis of the Procrustes analysis was matched with the reference data. When calculating the relative distance between two cars in relation to time, the actual distance between the two cars increases by the length of the car at a constant speed of $40 \mathrm{~km} / \mathrm{h}$, as shown in Figure. 8. As a result, the data generated by the correction algorithm shows the distance data between the two cars, and the actual numbers were calculated between 5.428 and 6.149 meters

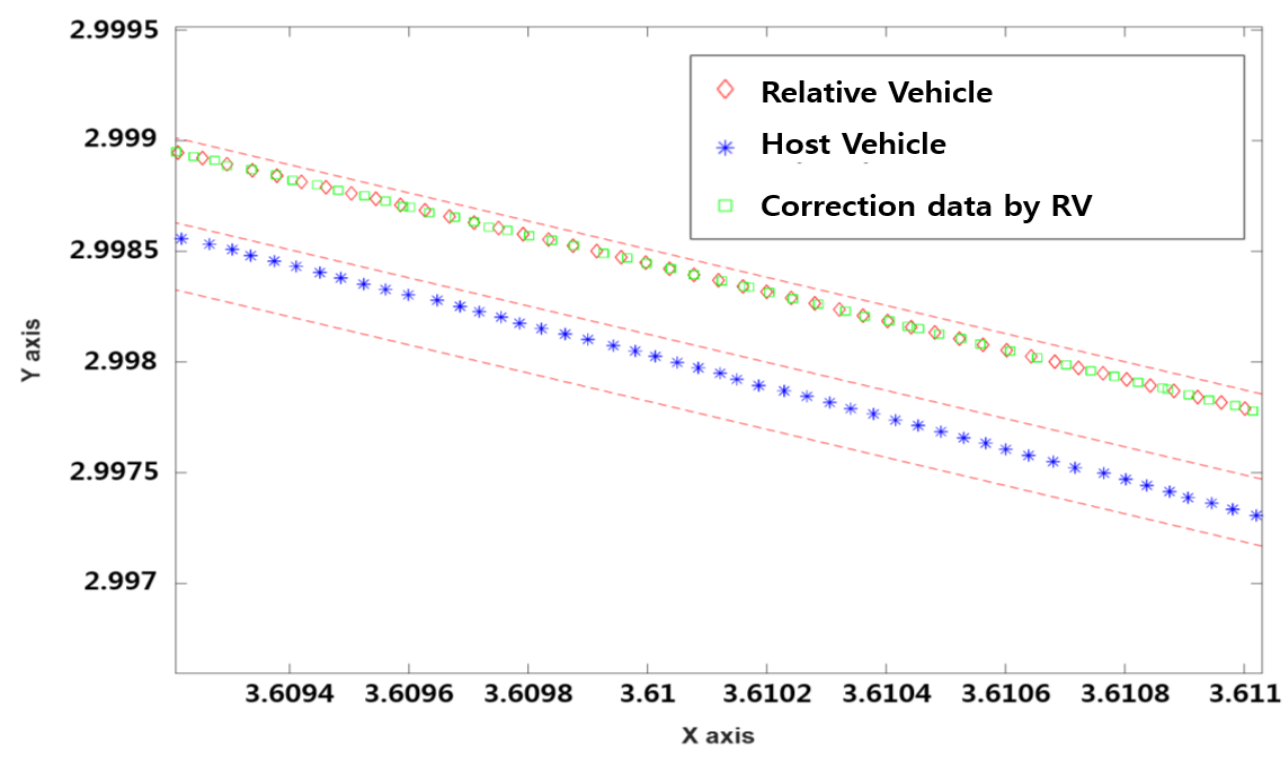

Figure 8. Result of Procrustes Algorithm (Straight)

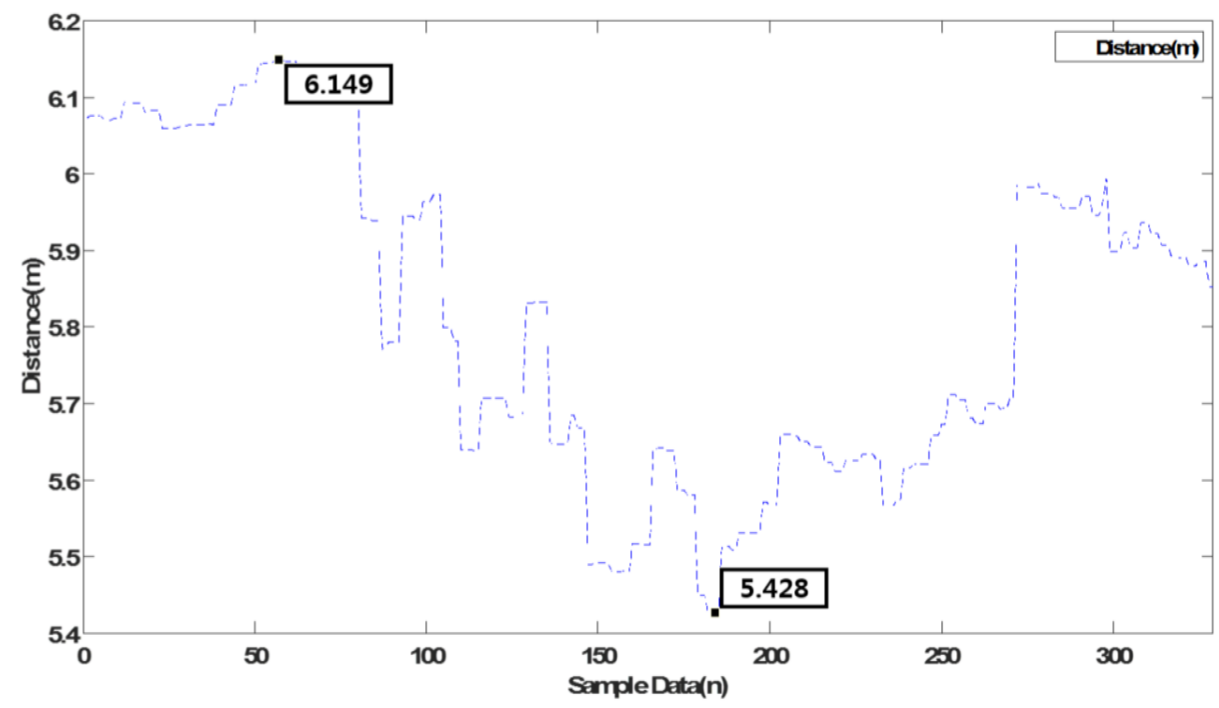

Figure 9. Correction by Procrustes Algorithm (Straight) 
4.2.2. Experimental results using video information and Procrustes analysis: In a straight road of $552 \mathrm{~m}$, two cars were positioned with 2-3 meters of space and were driven single file in speeds between $30 \mathrm{~km} / \mathrm{h}$ and $40 \mathrm{~km} / \mathrm{h}$. The GIS lane data were provided by the Research Center and were compared with the obtained data. A single Procrustes Analysis was used to match and improve the positional data between the two cars. As shown in Figure8, the correction data is expressed when the basis of the Procrustes analysis was matched with the reference data. When calculating the relative distance between two cars in relation to time, the actual distance between the two cars increases by the length of the car at a constant speed of $40 \mathrm{~km} / \mathrm{h}$, as shown in Figure. 8. As a result, the data generated by the correction algorithm shows the distance data between the two cars, and the actual numbers were calculated between 5.428 and 6.149 meters

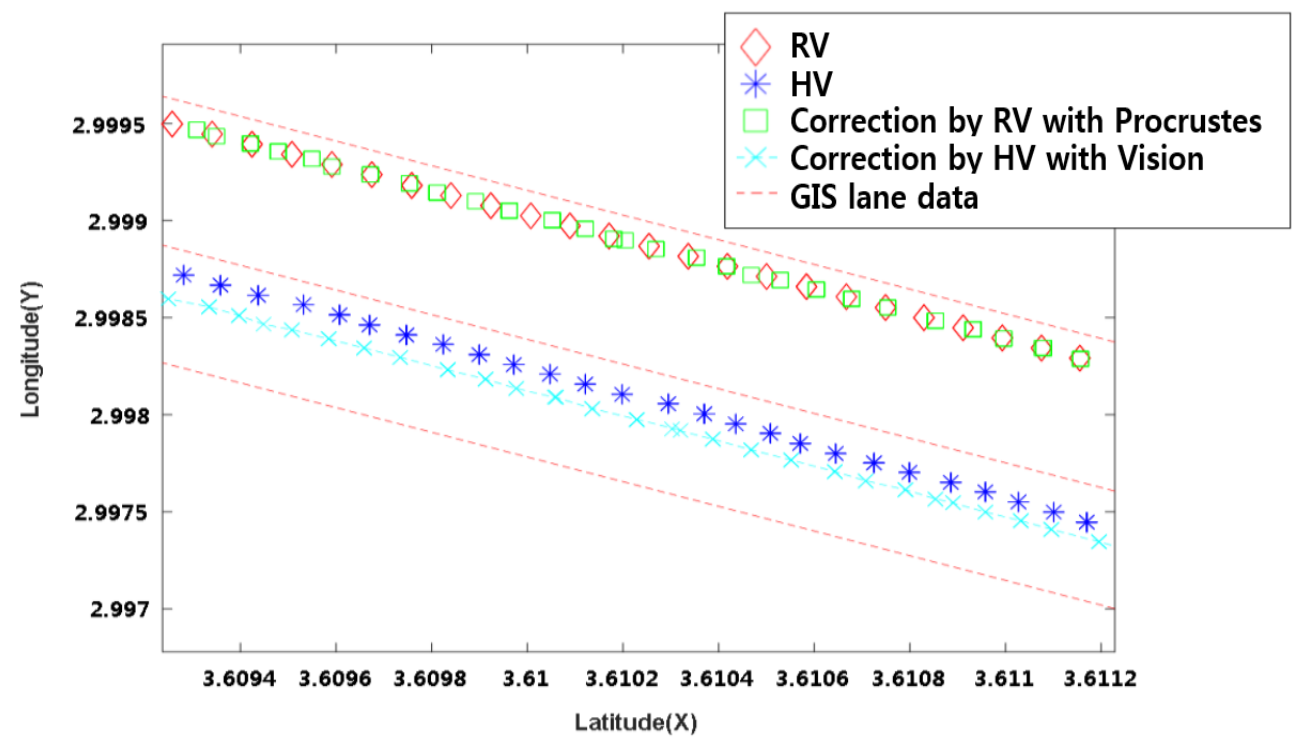

Figure 10. The Correction for HV Position by Lane Detector (Straight)

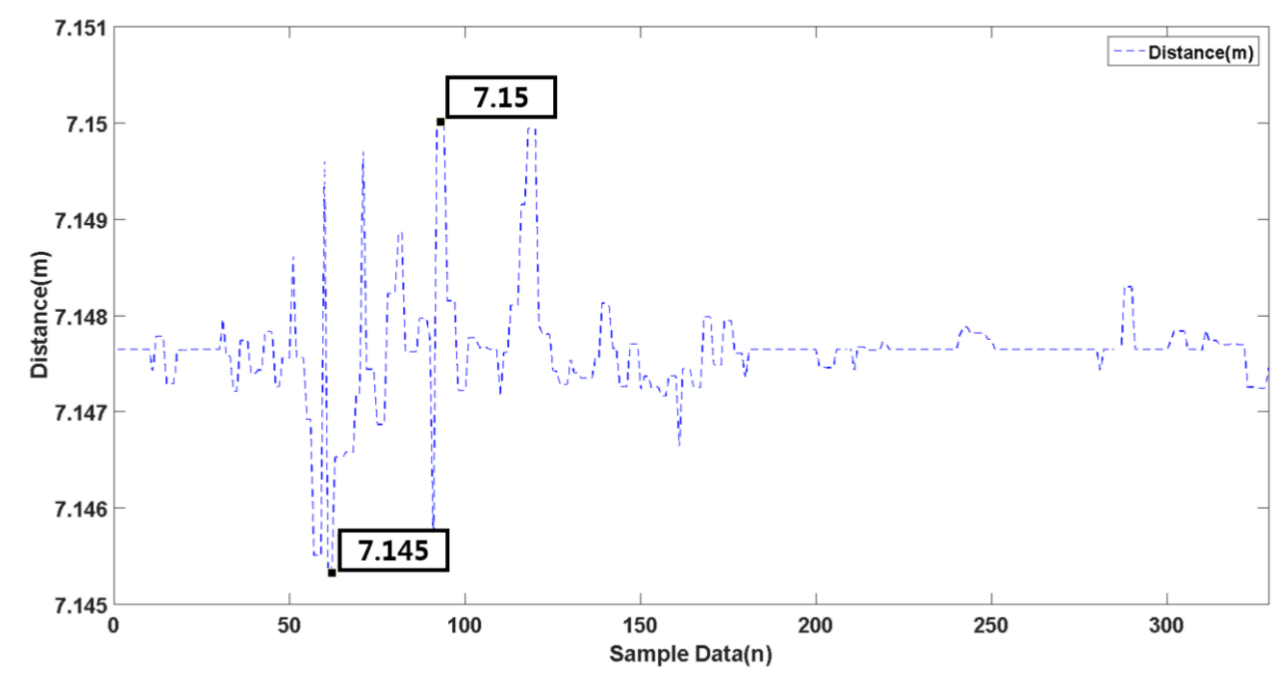

Figure 11. Vehicle Distance by Lane Data and Procrustes Algorithm

The positioning data matched from the Procrustes analysis based on the nearby car can also be compared, matched, and improved with the data from the video module. Figure. 9 presents the corrected driver car's data matched with the video 
data. The positioning data of the driver's vehicle and the GIS lane information shows a difference of 1.2 meters of the lanes, while the video data shows no difference when calculated.

The distance between the two cars is shown in Figure 9. Figure 10(a) shows the distance of the two cars, with Procrustes-corrected positioning data. Results in Figure 10(a) shows the point-to-point difference of positional data between the two cars; however, despite the constant distance in the single-file driving experiment, the results show a maximum distance of $6.149 \mathrm{~m}$ and a minimum distance of $5.428 \mathrm{~m}$, with an range of error of 0.721 . In contrast, positional data corrected by the Procrustes algorithm and matched with the video data are shown in Figure 10(b). The results show a maximum distance of 7.15 meters and a minimum distance of 7.14 meters, with a range of error of $0.005 \mathrm{~m}$. This shows a $99 \%$ improvement in accuracy from $0.721 \mathrm{~m}$ to $0.005 \mathrm{~m}$

\section{Conclusion}

These researches demonstrate a method to improve positioning accuracy based on the location information of vehicles and WAVE communication. The corrected coordinates were used to measure the distance between two cars. The driver vehicle's position was corrected and matched using the distance with a nearby car obtained from a low-cost lanedetection module. It was possible to obtain more precise positioning data by assisting the calculations with low-budget GPS and lane detection modules. In addition, the potential of V2V communications for improving positional accuracy was demonstrated.

This research concludes that more accurate results can be obtained with more vehicular data. This research can be expanded to real-road situations and roads with large curvature radii. A more comprehensive positioning correction algorithm can be obtained by adding in nearby car's visual data on top of vehicle data and GPS data.

\section{Acknowledgments}

This research was supported 100 -year-old elderly-friendly society corresponding product research and development project funded by the Department of Health and Human Services (No.HI15C1027)

\section{References}

[1] I. S. Weon, S. G. Lee and S. C. Moon, "Relative position obtained using the WAVE communication and verification algorithms for position correction", Asia-pacific Proceedings of Applied Science and Engineering for Better Human Life, vol. 7, (2016), pp. 44-47.

[2] S. C. Moon, S. G. Lee, D. H. Kim, I. S. Weon and S. G. Lee, "Classification of Roadway Type and Estimation of a Road Curvature Using a Road Characteristic Conversion Coefficient", Advanced Science and Technology Letters, vol. 90, (2015), pp. 33-38.

[3] S. C. Moon, S. G. Lee, J. J. Kim and B. S. Kim, "Detecting Lane Departure Based on GIS Using DGPS", Transaction of the Korean Society of Automotive Engineers, vol. 20, no. 4, (2012), pp. 16-24.

[4] K. Huber, F. Heuberger, C. Abart, A. Karabatic, R. Weber and P. Berglez, "PPP: Precision Point Positioning - Constraints and Opportunities", FIG Congress 2010, Facing the Challenges-Building the Capacity Sydney, (2010), pp. 11-16.

[5] S. Godha and M. E. Cannon, "GPS/MEMS INS integrated system for navigation in urban areas, GPS Solutions", Springer-Verlag, Berlin, Germany, vol. 11, no. 3, (2007), pp. 193-203.

[6] W. Wang and D. Wang, "Land vehicle navigation using odometry/INS/vision integrated system", IEEE International Conference on Cybernetics and Intelligent Systems, IEEE, (2008), pp. 754-759.

[7] A. Vu, A. Ramanandan, A. Chen, J. A. Farrell and M. Barth, "Real-time computer vision/DGPS-aided inertial navigation system for lane-level vehicle navigation", IEEE Transactions on Intelligent Transportation Systems, vol. 13 no. 2, (2012), pp. 899-913.

[8] S. B. Kim, S. Y. Lee, T. H. Hwang and K. H. Choi, "An advanced approach for navigation and image sensor integration for land vehicle navigation", Proceedings of 2004 IEEE 60th Vehicular Technology Conference, IEEE, vol. 6, (2004), pp. 4075- 4078. 
[9] S. C. Kim, "An evaluation of the performance wireless network in vehicle communication environment", The Journal of Korean Institute of Communications and Information Sciences, vol. 36, no. 10A, (2011), pp. 816-822.

[10] S. B. Yu, "Development of UTIS/WAVE Based Communication Technology; Vehicle Safety Services for Urban Area", Auto Journal: Journal of the Korean Society of Automotive Engineers, vol. 36, no. 7, (2014), pp. 33-39.

[11] I. S. Won, S. G. Lee, S. C. Moon and S. K. Lee, "Development of Satellite info Exchange Subsystem for Precision Positioning based on WAVE Communication", The Conference of Korea Society for Precision Engineering, (2015), pp. 76-77.

[12] F. Muller, D. Tapia and M. Kranz, "Precise Relative Positioning of Vehicles with On-the-Fly Carrier Phase Resolution and Tracking", International Journal of Distributed Sensor Networks, no. 8, (2015), p. 11.

[13] P. S. Heo, "A Study on the Automotive ADAS Market Diffusion Factors", "Proceedings of KICS", (2009), pp. 942-945.
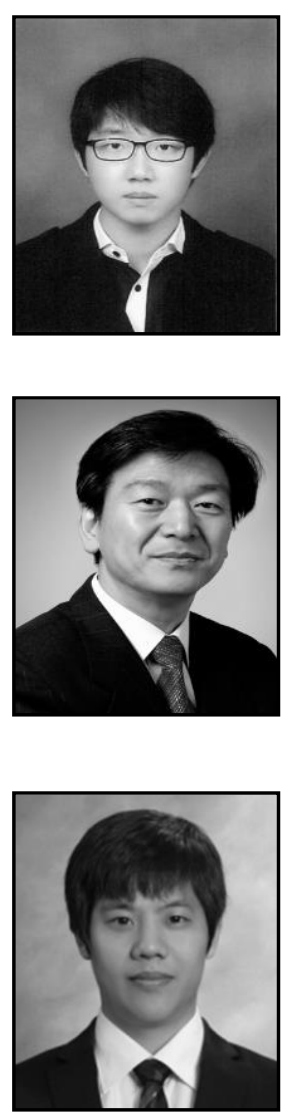

\section{Authors}

Ihn Sik Weon, he is a Master and Doctor of unify course in Department of Mechanical Engineering, KyungHee University, Korea. He has done his B.S in Control and instrument engineering from Korea University. Presently he is carrying his research work as development of position accuracy for autonomous driving and control vehicle, mobility system.

Soon Geul Lee, he is a professor in Department of Mechanical Engineering, KyungHee University, Korea. He has done his Ph.D in Human Engineering from University of Michigan. His areas of specialization are Control mobility system, balancing robot and mobility system by CMG and Detection environment indoor and outdoor for autonomous driving.

Sang Chan Moon, he is a Researcher in Core Tech R\&D Lab., LIGNex1, Korea. He has done his Ph.D in Mechanical Engineering from Kyung Hee University. $\mathrm{He}$ is in interested in autonomous driving, Precision positioning of vehicle, recognition behavior and learning algorithm. Presently his areas of specialization are Inertial navigation system, hybrid navigation for precision guidance system 